\title{
DIABETES MELLITUS TIPO 1: POSIBLE RELACIÓN CON LA INTERRUPCIÓN PRECOZ DE LA LACTANCIA MATERNA ${ }^{1}$
}

\author{
DIABETES MELLITUS TYPE 1: POSSIBLE RELATIONSHIP WITH EARLY \\ WEANING OF BREAST FEEDING \\ Dalila Teixeira Leal'2, Iêda Maria Ávila Vargas Dias ${ }^{3}$, Flávia Andrade Fialho \\ Lílian do Nascimento ${ }^{5}$, Patrícia Motta das Neves ${ }^{6}$, Maria José Guedes Gondim Almeida ${ }^{7}$
}

\section{RESUMEN}

Introducción: La dieta es importante en la patogénesis de la diabetes tipo 1 , los estudios indican una fuerte asociación entre la exposición temprana a la leche de vaca y la aparición de esta enfermedad. Objetivo: Verificar la existencia de la relación entre el destete precoz de la lactancia materna, la exposición a otras leches antes de seis meses de vida y el desarrollo de diabetes mellitus tipo 1. Materiales y Métodos: Investigación cuantitativa, a los padres respecto a los niños y adolescentes con diabetes tipo 1 que se registraron en el Departamento de Control de la Presión Arterial, Diabetes y Obesidad del Departamento Municipal de Salud de Juiz de Fora com una muestra total de 89 participantes. El instrumento utilizado para la recolección de datos fue un cuestionario estructurado aplicado a estos padres, los sujetos de la investigación fueron de ambos sexos y de todas las razas. Cabe señalar que el origen de esta investigación son los usuarios de los servicios de Diabetes Mellitus tipo 1, pero sólo los padres o tutores fueron entrevistados. Resultados: Se presentan datos sobre la caracterización de la muestra, la aparición o no de destete precoz de la lactancia materna, período de la lactancia materna exclusiva, si se ofreció dieta después del destete y las razones que llevaron a esta ocurrencia. Discusión y Conclusiones: La relación entre el destete precoz de la lactancia materna, con la consiguiente introducción de sustitutos de la leche

\section{ABSTRACT}

Introduction: Diet is important in the pathogenesis of tipe 1 diabetes, studies show a strong association between early exposure to cow's milk and the onset of the disease. Objective: To verify the existence of the relationship between early weaning of breast feeding and subsequent exposure to breast milk substitutes before six months of life with the development of type 1 diabetes mellitus. Materials and Methods: Quantitative research, which was to subject parents of children and adolescents with type 1 diabetes who were registered at the Department of Control of Hypertension, Diabetes and Obesity Municipal Health Department of Juiz de Fora, a total sample of 89 participants. Instrument used for data collection was a structured questionnaire applied to these parents, research subjects, of both sexes and all races. It is noteworthy that the origins of this research are service users of type $1 \mathrm{DM}$ patients, but only parents or guardians were interviewed. Results: Data are presented concerning the characterization of the sample; occurrence or not of early weaning of breast feeding, period of exclusive breastfeeding, offered diet after weaning and reasons that led to this occurrence. Discussion and Conclusions: The relationship between early weaning of breast feeding, with the consequent

1 Artículo Original de Investigación.

2 Enfermera. Facultad de Enfermería, Universidad Federal de Juiz de Fora, Minas Gerais, Brasil.

3 Enfermera. Doctora en Enfermería. Profesora del Departamento de Enfermería y del Programa de Póst-Graduación en Enfermería, de la Universidad Federal del Amazonas / UFAM. Líder del Grupo de Investigación en Enfermería - Salud de la Mujer, del Recién-Nacido, Niño y Adolescente de la Universidad Federal del Amazonas / UFAM. Manaus, AM, Brasil.

${ }_{4}$ Enfermera. Magíster en Enfermería. Doctoranda en Salud Colectiva y Bioética, Fundación Oswaldo Cruz (Fiocruz), Rio de Janeiro, Brasil. Miembro del Grupo de Investigación en Enfermería - Salud de la Mujer, del Recién Nacido, Niño y Adolescente de la Universidad Federal del Amazonas (UFAM). Correspondencia: Santos Dumont No. 128/303 - Centro / Juiz de Fora / MG - CEP: 36010-510.Tel: (32) 3216-2299. E-mail: flavinhafialho@bol.com.br

5 Enfermera. Maestrante en Enfermería, Universidade Federal de Juiz de Fora (UFJF-MG) Brasil.

${ }^{6}$ Enfermera. Especialista en Enfermería Neonatal, Unión San Camiliana / Facultad de Enfermería Luisa de Marillac, Juiz de Fora, Brasil.

${ }_{7}$ Médica. Profesora de la Facultad de Derecho de la Universidad Federal de Juiz de Fora / UFJF. Maestra de Ginecología, Obstetricia y Mastología de la Universidad Estadual Paulista / USP, São Paulo, Brasil.

Artículo recibido el 2 de Octubre de 2012 y aceptado para publicación el 26 Noviembre de 2012. 
materna, podría conllevar al desarrollo de diabetes mellitus tipo 1. (Rev Cuid 2012; 3(3):293-9).

Palabras clave: Diabetes Mellitus Tipo 1. Lactancia Materna. Enfermería. (Fuente: DeCS BIREME).

\section{INTRODUCCIÓN}

La discusión sobre la lactancia materna (LM) ha venido mostrando en los últimos años en relación a su importancia, el cumplimiento y los factores que influyen en su decisión y su duración. De acuerdo con los criterios de la Organización Mundial de la Salud (OMS), la lactancia materna exclusiva hasta los seis meses de vida del bebé, complementada con otros alimentos hasta los dos años de vida es ideal y ayuda a reducir el riesgo de anemia y la desnutrición de los niños, entre otros problemas de la Salud (1).

La evidencia de estudios epidemiológicos y experimentales demuestran que los factores ambientales juegan un papel importante en la diabetes tipo 1, la etiología de la que estaba vinculado tradicionalmente a factores genéticos, con pocas posibilidades de prevenir su aparición. Estos estudios pretenden demostrar que, con la nueva comprensión de la patogénesis de la diabetes tipo 1, puede ser posible prevenir su aparición, además de la posibilidad de reducir sustancialmente sus efectos nocivos en las personas que ya sufren de la enfermedad (2).

La dieta es importante en la patogénesis de la diabetes tipo 1 , los estudios indican una fuerte asociación entre la exposición temprana a la leche de vaca y la aparición de esta enfermedad. Por lo tanto, los niños expuestos tempranamente a la leche de vaca tienen una mayor probabilidad de contraer la enfermedad en comparación con aquellos que recibieron lactancia materna exclusiva hasta por lo menos cuatro meses después de nacimiento (2). Así que la leche materna es un factor protector para la diabetes mellitus tipo 1 , debido a las propiedades antiinfecciosas de este tipo de leche, o el hecho de que la lactancia materna podría evitar que los niños podrían ser prematuros expuestos a otros agentes infecciosos contenidos en sucedáneos de la leche (3).

Además de estos beneficios físicos, el acto de la lactancia materna es también importante para las relaciones afectivas entre madre e hijo. Sin embargo, según la OMS la prevalencia de la lactancia materna exclusiva es baja, su duración es corta y la lactancia materna exclusiva hasta los seis meses de edad es rara, por lo tanto, se introducen alimentos complementarios pronto para una gran mayoría de los niños. Estos alimentos son a menudo deficientes en contenido de energía y nutrientes. En muchas familias, se preparan en condiciones higiénicas desfavorables, a veces almacenados a temperatura ambiente durante períodos prolongados, y casi universalmente son ofrecidos en biberón, especialmente en el primer año de vida (1). introduction of breast milk substitutes, may lead to the development of type 1 diabetes mellitus.

Key words: Diabetes Mellitus, Type 1. Breast Feeding. Nursing. (Source: DeCS BIREME).

Existen numerosos beneficios que la lactancia materna proporciona, y desde el punto de vista biológico, cada mujer es capaz de producir la cantidad y la calidad de leche necesaria para el buen desarrollo de su hijo ya que los niveles hormonales son adecuados y hay una retirada eficiente de la leche de mama. Reforzar las acciones en favor de la lactancia materna, los beneficios son evidentes para la mujer a través de esta práctica, tales como la reducción de la hemorragia posparto, a través de la contracción uterina, la anticoncepción, a condición de que la lactancia materna exclusiva se practica en la demanda, y la disminución debida a la anemia debido a retraso menstrual y reducir el riesgo de cáncer de mama y ovario (4)

Dada los innumerables esfuerzos para llevar a cabo este estudio, que tiene como objetivo determinar la existencia de una relación entre el destete temprano de la LM y su posterior exposición a sustitutos de la leche materna antes de los seis meses de vida con el desarrollo de diabetes mellitus tipo 1 .

\section{MATERIALES Y MÉTODOS}

El procedimiento de búsqueda del conocimiento es un proceso reflexivo, sistemático, controlado y crítico que permite descubrir nuevos hechos o datos, soluciones o leyes en cualquier área de conocimiento. Por lo tanto, la investigación es una actividad orientada a la solución de problemas mediante el método científico (5).

El método científico es visto como un método para lograr un mayor conocimiento y se ha utilizado de manera productiva por los investigadores que se dedican al estudio de una amplia gama de temas de interés para el desarrollo de una base científica para ciencia (6). En el método científico, hay dos enfoques para el análisis de datos: cualitativa y cuantitativa. Este último, por sus características serán empleados en el presente estudio.

La investigación cuantitativa consiste en la recogida sistemática de información numérica, por lo general en condiciones de mucho control, utilizando procedimientos estadísticos para el análisis. Este tipo de investigación tiende a enfatizar el razonamiento deductivo, las reglas de la lógica y los atributos mensurables de la experiencia humana. Se caracteriza por el énfasis en la objetividad en la recopilación y análisis de datos, lo cual se logra a través de procedimientos estructurados e instrumentos formales (6). Este proyecto en su primera fase solicitó la autorización del Servicio Municipal de Salud (SMS) en la ciudad de Juiz de Fora para llevar a cabo la recogida de datos y remisión de la misma al Comité de Ética de la Universidad Federal de Juiz de Fora, para la debida 
aprobación, después de haber cumplido con las normas para la realización de Investigación en Seres Humanos, la Resolución 196/96, el Consejo Nacional de Salud, 10/10/1996. Tal como fue aprobado por el Comité de Ética con el dictamen $n^{\circ} 274 / 2007$.

Poco después, se llevó a cabo en un niño insulinodependiente del Servicio de Control de Hipertensión, Diabetes y Obesidad del Adolescente (SCHDO) de la Salud Municipal de Juiz de Fora. El SCHDO es un servicio de atención secundaria responsable del cuidado de los pacientes de las Unidades Básicas de Salud del municipio, con los siguientes criterios de inclusión: diagnóstico de hipertensión nivel 3, grupo $\mathrm{C}$, difícil control de la hipertensión, diabetes mellitus tipo 1 , diabetes mellitus tipo 2 con complicaciones, obesidad grado 3 .

Después de establecer la atención, se inició el estudio piloto que sirve como indicador de los cambios necesarios que se realizaron a fin de facilitar la aplicación del instrumento de la encuesta. Después de la aplicación del instrumento se le dio la fase de continuación de la recopilación de datos que fue precedido por la firma del consentimiento informado.
El instrumento utilizado para la recolección de datos fue un cuestionario estructurado, administrado a los padres de niños y adolescentes con diabetes tipo 1, registrada en SCHDO, de ambos sexos y todas las razas, para una muestra total de 89 participantes. Cabe señalar que el origen de esta investigación son los usuarios de los servicios de DM tipo 1, pero sólo los padres o tutores fueron entrevistados.

Después de la recogida de los datos, se tabularon y se clasificaron sistemáticamente para ser presentados mediante parámetros estadísticos descriptivos. Estadística descriptiva, como su nombre lo indica, se ocupa de describir los datos, su objetivo es sintetizar una serie de valores de la misma naturaleza, lo que les permite tener una visión general de la variación de estos valores (7).

\section{RESULTADOS Y DISCUSIÓN}

Se presentan datos sobre la caracterización de los participantes, ocurrencia o no de un destete temprano de LM, período de lactancia materna exclusiva y la dieta ofrecida tras el destete y las razones que llevaron a esta ocurrencia.

\section{Tabla I - Características de los participantes}

\begin{tabular}{|ccc|}
\hline Descripción & Edad (años) & Peso al nacer $(\mathbf{K g})$ \\
\hline Promedio & 13,35 & 2,95 \\
\hline Desviación Standard & 5,09 & 0,71 \\
\hline Mínimo & 4 & 1,0 \\
\hline Máximo & 27 & 4,2 \\
\hline
\end{tabular}

La Tabla I muestra que, en términos de edad, hubo un rango de 4 a 27 años con una media de 13,35 años. Es conveniente aclarar que una persona con 27 años, se incluyó en el estudio por ser usuario del servicio desde la infancia. Para el peso al nacer, se observó una variación de $1,0 \mathrm{~kg}$ a 4,2 kg, con una media de 2,95 kg.

\section{Tabla II - Nivel de educación de los padres}

\begin{tabular}{|lcc|}
\hline \multicolumn{1}{|c|}{ Variable } & Frecuencia & Porcentaje (\%) \\
\hline 1er grado completo & 7 & 8 \\
\hline 1er grado incompleto & 55 & 62 \\
\hline 1er grado en curso & 1 & 1 \\
\hline 2do grado completo & 18 & 20 \\
\hline 2do grado en curso & 1 & 1 \\
\hline 3er grado completo & 3 & 4 \\
\hline 3er grado incompleto & 2 & 2 \\
\hline Postgrado & 2 & 2 \\
\hline Total & $\mathbf{8 9}$ & $\mathbf{1 0 0 , 0}$ \\
\hline
\end{tabular}


En relación a la escolaridad, es de destacar que entre los 89 participantes que respondieron, 55 (62\%) no tenían una escuela primaria y sólo $3(4 \%)$ tuvieron el tercer grado. La educación en diabetes es una parte esencial de la atención al paciente, junto con un adecuado control metabólico, la actividad física y la dieta. Una mejor calidad de vida está estrechamente relacionada con el nivel más alto de conocimiento sobre la enfermedad y sus complicaciones. La explicación conduce a una reducción en el número de episodios de hipoglucemia, menos hospitalizaciones, mejor control metabólico y una mayor aceptación de la enfermedad (8). El nivel de la educación puede ser un importante facilitador del proceso de educación para la salud.

En cuanto a los ingresos familiares, a partir de 89 familias, $45(51 \%)$ de ellos viven con un ingreso familiar de hasta tres salarios mínimos, 32 (36\%) viven con un ingreso por debajo del nivel de pobreza; 10 (11\%) de las familias tenía un ingreso de hasta cinco salarios mínimos y 2 (2\%) de las familias tenían un ingreso de más de diez veces el salario mínimo. Este es un hecho que no se puede evaluar por separado, cuando se habla de los ingresos familiares es importante que se hace en relación con el número de dependientes.

Los estudios demuestran que existe una estrecha relación entre el ingreso familiar y el control de la diabetes, cuanto menor sea el ingreso mayor es la dificultad en el manejo de la enfermedad (9). Hecho significativo en nuestro estudio, ya que la mayoría de los encuestados tienen ingresos de hasta tres salarios mínimos. Tal relación puede interferir con la obtención de herramientas y equipos para controlar la enfermedad. Dado que, debido a que es una enfermedad crónica, su tratamiento implica medicamentos, atención especializada, entre otros.

Tabla III - Destete precoz de LM

\begin{tabular}{|ccc|}
\hline Variable & Frecuencia & Porcentaje (\%) \\
\hline Sí & 61 & 68 \\
\hline No & 22 & 25 \\
\hline No sabe & 6 & 7 \\
\hline Total & 89 & 100 \\
\hline
\end{tabular}

Para la muestra considerada destete precoz de la LM, se encontró en 61 individuos que representan el $68 \%$ de la muestra, mientras que $22(25 \%)$ de los niños fuente de investigación, de acuerdo con el relato de los padres recibieron lactancia materna exclusiva hasta los seis meses de edad, seis $(7 \%)$ de los participantes no pudieron informar sobre el período de lactancia materna exclusiva.

Cabe destacar que en este estudio, el destete precoz de la LM se define de acuerdo a las directrices del Ministerio de Salud, es decir, el niño ha sufrido el destete precoz de la LM y ya no recibe exclusivamente leche materna antes de los seis meses (10). Estos datos informan aspectos importantes de la lactancia materna, especialmente que un alto porcentaje de los niños tenía ocurrencia histórica de destete precoz. Esto nos permite mostrar una relación significativa entre el destete precoz y la posterior exposición a la leche materna sustituta antes de seis meses de vida con el desarrollo de diabetes mellitus tipo 1 .

Los estudios muestran que la leche materna y la leche de vaca, respectivamente, son un factor de protección y de riesgo para la diabetes mellitus tipo1 (3). La exposición temprana a la leche de vaca puede ser un determinante importante de esta condición. Muchos casos podrían evitarse si los niños de hasta 3 meses no recibieran leche de vaca, ya que existen pruebas de una relación entre la introducción temprana de alimentos complementarios para el desarrollo de enfermedades atópicas (11).

Para evitar la exposición temprana de los infantes a los componentes de la leche de vaca pueden también evitar se las condiciones favorables para la aparición de la diabetes mellitus, tipo 1 (12). Esta enfermedad puede ser provocada por el consumo de fórmulas iniciales basadas en leche de vaca, como albúmina de suero bovino (BSA) que es responsable de la destrucción de las células pancreáticas, lo que conduce a las condiciones para el desarrollo de la enfermedad. En este sentido, la introducción correcta de los alimentos debe hacer más hincapié en los programas para promover la lactancia materna, sobre todo entre las madres más jóvenes y pertenecientes a los grupos socioeconómicos más desfavorecidos (13). 
Tabla IV - Edad para el destete de LM

\begin{tabular}{|ccc|}
\hline Variable & Frecuencia & Porcentaje (\%) \\
\hline Antes de 1 mes & 61 & 39 \\
\hline Hasta 1 mes & 22 & 11 \\
\hline 2 meses & 6 & 13 \\
\hline Hasta 3 meses & 89 & 15 \\
4 meses & 61 & 3 \\
\hline Hasta 5 mes & 22 & 2 \\
\hline 6 meses & 6 & 7 \\
\hline Más de 6 meses & 89 & 100,00 \\
\hline Total & 89 & 10 \\
\hline
\end{tabular}

Sólo $6(10 \%)$ niños fueron amamantados durante más de 6 meses. Del total de 61 niños $(68 \%)$ son destetados precozmente. De ellos 24 (39\%) fueron destetados antes de su primer mes de vida.

Teniendo en cuenta el alto porcentaje de niños que fueron destetados precozmente, se observa la importancia de la lactancia materna durante el primer mes de vida y pone de manifiesto la relevancia del estudio, ya que los antecedentes de estos niños, que ahora viven con diabetes mellitus tipo 1 , muestra que no han tenido una lactancia materna exclusiva hasta los seis meses de edad. Vale la pena mencionar que, además de los hallazgos de destete precoz, también se encontró un déficit en todo el proceso de la alimentación, ya que esto podría haber sido mantenido hasta los dos años de vida y se complementa con otros alimentos, lo que no ocurrió.

Los estudios muestran la relación entre el destete precoz de la LM y la consiguiente introducción de sustitutos de leche materna con el desarrollo de diabetes mellitus tipo 1 (14-16). Por lo tanto, los profesionales de la salud deben crear estrategias que mejoren la eficacia de la lactancia materna en este periodo, ya que se recomienda que la lactancia materna es exclusiva hasta los 6 meses y complementada con otros alimentos hasta los 2 años de edad o más.

Tabla V - Dieta ofrecida después del destete de LM

\begin{tabular}{|cc|}
\hline Variable & Frecuencia \\
\hline Leche de Vaca & 41 \\
\hline Leche industrializada & 24 \\
\hline Leche de vaca diluida & 17 \\
\hline Leche de cabra & 2 \\
\hline Avena & 4 \\
\hline Otro & 3 \\
\hline
\end{tabular}


Los alimentos utilizados después del destete fueron: leche entera de vaca, seguido por la fórmula de leche, leche de vaca y leche de cabra menos diluido y la avena, respectivamente. A mediados del siglo XIX, una gran cantidad de investigaciones buscaron un sustituto de la leche materna que se utilizará durante el período de destete; el resultado de estas investigaciones, muchas impulsadas por los intereses de la industria alimentaria, condujo al establecimiento de la lactancia artificial. Profesionales de la salud consideraron que al ofrecer sustitutos de la leche materna, proporcionaría una mejor nutrición para los niños. Esta práctica ha sido aceptada por muchas madres, lo que resulta en un cambio de comportamiento de estas mujeres a preferir la leche en polvo (17). La comprensión, aunque equivocada, parece utilizarse en la práctica de la lactancia materna hasta el día de hoy.

Mediante el estudio de la duración de la lactancia materna después de la introducción de otra leche en la alimentación infantil se observa que la leche de vaca, líquido o en polvo, formulado o no, es un competidor de la leche materna y que su introducción marca el inicio del proceso de destete. La introducción de otra leche después de la alimentación está bien establecida, como es el caso de los niños mayores, que parece tener menos influencia en la reducción de la duración de la lactancia materna (18). Por lo tanto, parece razonable deducir que, como más adelante se introdujeron otros alimentos, serán más las madres que tienden a amamantar a su hijo. En cuanto a la razón para el destete, 28 (32\%) informaron que se produjo por la voluntad de su madre, $19(21 \%)$ reportaron haber sido por cese de la producción de leche, $14(16 \%)$ por condiciones médicas maternas, 11 (12\%) condición clínica del niño, 9 (10\%) se justifica destete porque tienen leche débil, $5(6 \%)$ reportaron ocupaciones y $3(3 \%)$ reportaron lesión de mama como causa de destete precoz. Se observa en los resultados obtenidos que la voluntad de la madre fue predominante en el proceso de destete. En este sentido se postula, que tal vez existan factores culturales que se construyen socialmente para la continuación de la lactancia materna exclusiva, tanto para el niño como para la madre, que a menudo no conocen los beneficios de esta práctica.

Un dato de los resultados presentados, que merece ser aclarado es el hecho de que los participantes habían designado destete "producción de leche débil", se hace énfasis en que esto es un mito, porque fisiológicamente esta posibilidad no existe.

Por último, teniendo en cuenta que la aparición de destete se asocia con temas culturales y educativos, la enfermera juega un papel importante en la promoción y mantenimiento de la lactancia materna.

\section{CONCLUSIONES}

Las ventajas de la lactancia materna son muchas y reconocidas, ya sea en el corto o largo plazo; existe un consenso global de que su práctica exclusiva es la mejor forma de alimentar a los bebés hasta los seis meses de vida. De los resultados de este estudio, se puede aludir a aspectos importantes indicando que la lactancia materna también podría ayudar en la prevención de la diabetes mellitus tipo 1. Desde que se descubrió una posible relación entre el destete precoz de la LM y la posterior exposición a sustitutos de la leche materna, antes de los seis meses de vida, con el desarrollo de esta patología.

Frente a esto, la práctica de la lactancia materna exclusiva hasta los seis meses de vida del niño, gana fuerza y su promoción debe ser una prioridad en las actividades de los enfermeros y otros profesionales de la salud. Una estrategia importante para fomentar esta práctica es establecer bancos de leche. La Red Brasileña de Bancos de Leche Humana (HMB) tiene las siguientes funciones: promover, proteger y apoyar la lactancia materna, distribuir leche humana de calidad certificada, contribuir a la reducción de la mortalidad infantil, unir esfuerzos para el Pacto Nacional para la Reducción de la Mortalidad Materna y Neonatal. En este sentido, la red desarrolla un trabajo para ayudar a las mujeres-madres durante la lactancia, ya que también capacita y asesora sobre la salud infantil.

Algunas mujeres en período de lactancia cuando producen un volumen de leche más allá de la necesidad del bebé, permite a los donantes a ser un Banco de Leche Humana. De acuerdo con la legislación que regula el funcionamiento de bancos de leche en Brasil (Resolución del Directorio $N^{\circ} .171$ de 4 de septiembre de 2006) el donante, además de presentar secreción láctica superior a las necesidades de su hijo, estar sano y estar dispuestos a donar los excedentes de la leche.

La leche materna es sin duda el mejor alimento para el recién nacido, en la composición nutricionalmente equilibrada, temperatura adecuada, ser estéril, tiene diferente composición de acuerdo con la maduración de las mamas y la capacidad del estómago del niño, permite una relación madre / hijo armoniosa y no ofrece prácticamente ninguna reacción alérgica. Además, tiene ventajas de orden económico. Asegurando así el alimento ideal para el bebé, ya que garantiza un crecimiento y desarrollo saludable. Por lo tanto, las enfermeras y otros profesionales de la salud tienen un papel importante en la promoción de la lactancia materna, pueden fomentar este acto a través de la sensibilización, la educación, el trabajo directo con el público, en grupos educativos y especialmente a las madres para ayudar en el desarrollo de la práctica de la lactancia materna.

En este proceso de educación es importante involucrar a toda la familia y evitar el uso de lenguaje y comunicación rígida, ya que muchas veces los mensajes dirigidos a las mujeres, en relación con el valor y la importancia de la lactancia materna, tienen un enfoque superficial, apelando a los sentimientos e instinto maternal. En los mensajes de las campañas en general no se abordan las posibles complicaciones y dificultades de no ofrecer lactancia materna, en especial para el recién nacido. Esto no constituye una garantía para el éxito absoluto de la lactancia materna. Corresponde al profesional de la salud, aclarar y apoyar a las mujeres a realizar 
esta práctica. Las enfermeras y otros profesionales de la salud deben estar capacitados para informar de las consecuencias del destete precoz de la lactancia materna, por lo que puede ayudar a lograr el objetivo diseñado por la Organización Mundial de la Salud (OMS), ya que incluso con el Programa Nacional para fomentar la lactancia materna, la tasa de lactancia materna exclusiva hasta el sexto mes es todavía baja en Brasil.

Los resultados de este estudio corroboran esta afirmación, ya que la mayoría de los sujetos de la investigación fueron sometidos a destete precoz de LM.

Teniendo en cuenta estos resultados, es evidente la importancia de realizar nuevos estudios. Sería interesante promover el debate y reflexionar sobre los resultados que fueron importantes en este estudio, por lo que requiere más investigación con este tema, especialmente investigación con buen rigor metodológico.

Por último, se activa la discusión sobre la importancia de la lactancia materna en relación con el cumplimiento y factores que influyen en la decisión de amamantar o no, así como la necesidad de trabajar más para promover la lactancia materna, especialmente, hasta los primeros seis meses de vida del niño, ya que se cree que con la aplicación de esta práctica se puede prevenir la introducción temprana de otros alimentos en la alimentación infantil, que como se evidencia en este estudio puede estar asociada con la aparición de la diabetes mellitus tipo 1 y otras enfermedades.

\section{REFERENCIAS BIBLIOGRÁFICAS}

1. Ministerio de Salud. Organización Panamericana de la Salud. Guia de alimentos para niños menores de dos años. Brasília: Ministerio de Salud, 2002.

2. Medeiros JS, Rivera MAA, Benigna MJC, Cardoso MAA, Costa MJC. Case-control sobre la exposición temprana a la leche de vaca y la incidencia de diabetes tipo 1 en Campina Grande, Paraíba. Rev. Bras Mater Infant Health . Julio 2003; 3 (3): 271-280.

3. Gimeno SGA, Souza JMP. La lactancia materna, leche de vaca y la diabetes mellitus tipo 1: examinar la evidencia. Rev Bras Epidemiol. Abril 1998 1; 1: 4-13.

4. Ramos RC, Almeida JAG. Las reclamaciones de destete materno: un estudio cualitativo. J Pediatr. Septiembre 2003; 79 (5): 385-390.

5. Rampazzo L. Metodología Científica - para estudiantes de carreras de grado y posgrado. Lorena: Stiliano, 1998.

6. Polit DF, Hungler BP. Fundamentos de la investigación en enfermería. 3a ed. Porto Alegre: Medical Arts, 1995.

7. Guedes TA, Acorsi CRL, Martins ABT, Jan V. Hacer el aprendizaje Docencia Estadística de diseño. 2005. Disponible en: http://www.each.usp.br/rvicente/Guedes_etal_Estatistica_Descritiva.pdf

8. Maia MGM, Neto JT, Rego RCF, Muniz PT . Los factores asociados con la interrupción de la lactancia materna en niños menores de seis meses de edad, de la ciudad de Río Branco (Acre). Ap Bahía de salud pública. 2006. Ene-june 30, 1: 129-140.

9. Silveira VMF, Menezes AMB, Post CLA, Machado CE. Una muestra de pacientes con diabetes tipo 1 en el sur de Brasil. Arq Bras Endocrinol Metab. Octubre 2001; 45(5): 433-440.

10. Organización Panamericana de la Salud. Lactancia Materna. 2003. Disponible en: http://www.opas.org.br/siste$\mathrm{ma} /$ fotos/amamentar.pdf

11. Monte CMG, Giugliani ERJ. Recomendaciones para la alimentación complementaria del niño amamantado. J Pediatr. Noviembre 2004; 8(5):131-141.

12. Silva IA. Reflexiones sobre la práctica de la lactancia materna. Ap Esc Enferm USP.Abril 1998; 1: 58-72.

13. Bueno MB, Souza JMP, Souza SB, Paz SMRs, Gimeno SGA, Siqueira AAF. Los riesgos asociados con el proceso de destete de los lactantes nacidos del Hospital Universitario de São Paulo entre 1998 y 1999: estudio de cohorte prospectivo del primer año de vida. Cad salud pública. Septiembre 2003; 5: 1453-1460.

14.Zanetti ML, Mendes IAC, Ribeiro PK. El reto para el control del hogar en niños y adolescentes con diabetes tipo 1. Rev Latinoam enferm. Julio 2001; 4: 32-36.

15. Damiani D. Diabetes mellitus tipo 1 y anticuerpos contra albúmina de suero bovino. Rev. Asoc. Med. Bras. Abril, Junio 2004; 50(2): 119.

16. Araújo MFM, Otto AFN, Schmitz BAS. Primera evaluación del cumplimiento de los "Diez Pasos para una Lactancia Exitosa" Hospitales Amigos de los Niños del Brasil. Rev. Bras Mater Infant Health. Octubre, Diciembre 2003; 3 (4): 411-419.

17. Bosi MLM, Machado MT. La lactancia materna: reseña histórica. Notebooks ESP. Julio- Diciembre 2005.

18. Bueno MB, Souza JMP, Paz SMRs, Souza SB, Cheung PPY, Augusto RA. Duración de la lactancia materna después de la introducción de otro tipo de leche: seguimiento de la cohorte de niños nacidos en un hospital universitario de Sao Paulo. Rev Bras Epidemiol. Agosto 2002; 2: 145-152. 